\title{
Strange quark matter in a chiral SU(3) quark mean field model
}

\author{
P. Wang, V. E. Lyubovitskij, Th. Gutsche, and Amand Faessler \\ Institut für Theoretische Physik, Universität Tübingen, Auf der Morgenstelle 14, \\ D-72076 Tübingen, Germany
}

\begin{abstract}
We apply the chiral SU(3) quark mean field model to investigate strange quark matter. The stability of strange quark matter with different strangeness fraction is studied. The interaction between quarks and vector mesons destabilizes the strange quark matter. If the strength of the vector coupling is the same as in hadronic matter, strangelets can not be formed. For the case of $\beta$ equilibrium, there is no strange quark matter which can be stable against hadron emission even without vector meson interactions.
\end{abstract}

PACS: 11.30.Rd; 12.39.Ki; 14.65.Bt

Keywords: Strange quark matter; chiral symmetry; relativistic mean field.

\section{INTRODUCTION}

Strange quark matter has attracted a lot of interest since Witten suggested that it could be absolutely stable even at zero temperature and pressure [四]. The investigation of such a possibility is relevant not only for high energy physics, but also for astrophysics. For example, the core of a neutron star may be composed of quark matter. The possible existence of strange stars which are made entirely of deconfined $\mathrm{u}, \mathrm{d}$ and s quarks is one of the most intriguing aspects of modern astrophysics. There have been some reports of events with $\mathrm{A} \simeq 350-500$ and $\mathrm{Z} \simeq 10-20$ in cosmic ray experiments [2]- [4], the so-called exotic cosmic ray events. Also, recent studies have shown that X-ray burst sources are likely strange star candidates [5]- [8]. It is also interesting to produce strange quark matter (strangelets) in the laboratory because they could serve as a signature of the formation of the quark-gluon-plasma which is a direct demonstration of QCD [9]- [11]. Many ultrarelativistic heavy-ion collision experiments at Brookhaven and CERN [12 are proposed to search for (meta)stable lumps of such kind of strangelets. Recently, Ardouin et al. [13] presented a novel method which can be applied to characterize the possible existence of a strange quark matter distillation process in heavy-ion collisions. Up to now, there is no experiment which confirms the existence of strangelets. For example, the E864 collaboration found that there is no evidence for strangelet production in $11.5 \mathrm{GeV} / \mathrm{c}$ per nucleon $\mathrm{Au}+\mathrm{Pb}$ collisions [14].

Besides the experimental efforts, there are also a lot of theoretical investigations of the stability of strange quark matter. The earliest discussions are based on the MIT bag model 
[15] which assumes that quarks are confined by a phenomenological bag. Within the bag quarks are asymptotically free. Calculations [16] within this model indicate that there is a range of parameters in which strange quark matter is absolutely stable, i.e. the energy per baryon is less than $930 \mathrm{MeV}$. The stability of strange quark matter with finite volume (strangelets) was also discussed in the MIT bag model. Berger and Jaffe [17] discussed the surface correction for the strangelets, where they found that the surface tension destabilizes strangelets. The curvature contribution was considered by Madsen [18] which is dominant for strangelets with small baryon numbers. Though the bag model is simple, it is an incomplete description of confinement. Results from lattice calculations [19] show that quark matter does not become asymptotically free and some hadronic degrees of freedom remain within the quark matter immediately after the phase transition. Fowler, Raha and Weiner 20] suggested another description of the confinement mechanism via the introduction of a density-dependent quark mass. This quark mass-density-dependent (QMDD) model was first employed to study the properties of ordinary quark matter [20] and then applied to the investigation of strange quark matter [21]- 25]. As was pointed in our recent paper 26], their thermodynamic treatment was not correct. We reconsidered strange quark matter in the self-consistent quark mass density dependent model and found a region of parameters in which the strange quark matter is absolutely stable.

In the QMDD model, the concept of a density dependent quark mass has no dynamical origin. In recent years, some approaches for strange quark matter based on dynamical models were developed. Alberico et al. [27] utilized the color dielectric model to calculate the energy per baryon of strange quark matter. They found that while the double minimum version of the color dielectric model allowed the existence of strangelets, the single minimum version of this model excluded the possibility. Stability of strange quark matter was also investigated using the effective 4-quark interactions [28], the SU(3) Nambu-Jona-Lasinio (NJL) model with and without 4-quark vector type interactions [29,30]. In studying hadronic matter, we proposed a chiral SU(3) quark mean field model. This chiral quark model was applied to investigate the properties of strange hadronic matter and multi-strange hadronic systems [31,32]. In this paper, we want to use this model to discuss the stability of strange quark matter. The difference between quark and hadronic matter is that in quark matter, the $\mathrm{u}$, d, s quarks are deconfined and not combined into baryons by the confining potential.

The paper is organized as follows. In section II, we introduce the basic model features. We apply the model to investigate strange quark matter in section III. The numerical calculations are discussed in section IV. Finally, main conclusions are drawn in section V.

\section{THE MODEL}

Our considerations are based on the chiral SU(3) quark mean field model (for details see Refs. [31,32]). For completeness, we introduce the main concepts of the model in this section. In the chiral limit, the quark field $q$ can be split into left and right-handed parts $q_{L}$ and $q_{R}: q=q_{L}+q_{R}$. Under $S U(3)_{L} \times S U(3)_{R}$ they transform as

$$
q_{L}^{\prime}=L q_{L}, \quad q_{R}^{\prime}=R q_{R}
$$

The spin- 0 mesons are written in the compact form 


$$
M\left(M^{+}\right)=\Sigma \pm i \Pi=\frac{1}{\sqrt{2}} \sum_{a=0}^{8}\left(\sigma^{a} \pm i \pi^{a}\right) \lambda^{a},
$$

where $\sigma^{a}$ and $\pi^{a}$ are the nonets of scalar and pseudoscalar mesons, respectively, $\lambda^{a}(a=$ $1, \ldots, 8)$ are the Gell-Mann matrices, and $\lambda^{0}=\sqrt{\frac{2}{3}} I$. Plus and minus sign correspond to $M$ and $M^{+}$. Under chiral SU(3) transformations, $M$ and $M^{+}$transform as $M \rightarrow M^{\prime}=L M R^{+}$ and $M^{+} \rightarrow M^{+^{\prime}}=R M^{+} L^{+}$. As for the spin-0 mesons, the spin-1 mesons are set up in a similar way as

$$
l_{\mu}\left(r_{\mu}\right)=\frac{1}{2}\left(V_{\mu} \pm A_{\mu}\right)=\frac{1}{2 \sqrt{2}} \sum_{a=0}^{8}\left(v_{\mu}^{a} \pm a_{\mu}^{a}\right) \lambda^{a} .
$$

They transform as $l_{\mu} \rightarrow l_{\mu}^{\prime}=L l_{\mu} L^{+}, r_{\mu}^{\prime}=R r_{\mu} R^{+}$. These matrices can be written in a form where the physical states are explicit. For the scalar and vector nonets, the expressions are

$$
\begin{gathered}
\Sigma=\frac{1}{\sqrt{2}} \sum_{a=0}^{8} \sigma^{a} \lambda^{a}=\left(\begin{array}{lcr}
\frac{1}{\sqrt{2}}\left(\sigma+a_{0}^{0}\right) & a_{0}^{+} & K^{*+} \\
a_{0}^{-} & \frac{1}{\sqrt{2}}\left(\sigma-a_{0}^{0}\right) & K^{* 0} \\
K^{*-} & \bar{K}^{* 0} & \zeta
\end{array}\right), \\
V_{\mu}=\frac{1}{\sqrt{2}} \sum_{a=0}^{8} v_{\mu}^{a} \lambda^{a}=\left(\begin{array}{lcc}
\frac{1}{\sqrt{2}}\left(\omega_{\mu}+\rho_{\mu}^{0}\right) & \rho_{\mu}^{+} & K_{\mu}^{*+} \\
\rho_{\mu}^{-} & \frac{1}{\sqrt{2}}\left(\omega_{\mu}-\rho_{\mu}^{0}\right) & K_{\mu}^{* 0} \\
K_{\mu}^{*-} & \bar{K}_{\mu}^{* 0} & \phi_{\mu}
\end{array}\right) .
\end{gathered}
$$

Pseudoscalar and pseudovector nonet mesons can be written in the same way.

The total effective Lagrangian for the description of strange quark matter is given by:

$$
\mathcal{L}_{\text {eff }}=\mathcal{L}_{q 0}+\mathcal{L}_{q M}+\mathcal{L}_{\Sigma \Sigma}+\mathcal{L}_{V V}+\mathcal{L}_{\chi S B}+\mathcal{L}_{\Delta m_{s}}+\mathcal{L}_{h}
$$

It contains the free part for massless quarks $\mathcal{L}_{q 0}=\bar{q} i \gamma^{\mu} \partial_{\mu} q$, the quark-meson field interaction term

$$
\mathcal{L}_{q M}=g_{s}\left(\bar{q}_{L} M q_{R}+\bar{q}_{R} M^{+} q_{L}\right)-g_{v}\left(\bar{q}_{L} \gamma^{\mu} l_{\mu} q_{L}+\bar{q}_{R} \gamma^{\mu} r_{\mu} q_{R}\right)
$$

the chiral-invariant scalar meson $\mathcal{L}_{\Sigma \Sigma}$ and vector meson $\mathcal{L}_{V V}$ self-interaction terms in the mean field approximation 31,33

$$
\begin{aligned}
\mathcal{L}_{\Sigma \Sigma}= & -\frac{1}{2} k_{0} \chi^{2}\left(\sigma^{2}+\zeta^{2}\right)+k_{1}\left(\sigma^{2}+\zeta^{2}\right)^{2}+k_{2}\left(\frac{\sigma^{4}}{2}+\zeta^{4}\right)+k_{3} \chi \sigma^{2} \zeta \\
& -k_{4} \chi^{4}-\frac{1}{4} \chi^{4} \ln \frac{\chi^{4}}{\chi_{0}^{4}}+\frac{\delta}{3} \chi^{4} \ln \frac{\sigma^{2} \zeta}{\sigma_{0}^{2} \zeta_{0}}, \\
\mathcal{L}_{V V}= & \frac{1}{2} \frac{\chi^{2}}{\chi_{0}^{2}}\left(m_{\omega}^{2} \omega^{2}+m_{\rho}^{2} \rho^{2}+m_{\phi}^{2} \phi^{2}\right)+g_{4}\left(\omega^{4}+6 \omega^{2} \rho^{2}+\rho^{4}+2 \phi^{4}\right),
\end{aligned}
$$

where $\delta=6 / 33 ; \sigma_{0}, \zeta_{0}$ and $\chi_{0}$ are the vacuum values of the mean fields $\sigma, \zeta$ and $\chi$ and the three terms $\mathcal{L}_{\chi S B}, \mathcal{L}_{\Delta m_{s}}$ and $\mathcal{L}_{h}$ which explicitly break the chiral symmetry. Chiral symmetry requires the following basic relations for the quark-meson coupling constants: 


$$
\begin{gathered}
\frac{g_{s}}{\sqrt{2}}=g_{a_{0}}^{u}=-g_{a_{0}}^{d}=g_{\sigma}^{u}=g_{\sigma}^{d}=\ldots=\frac{1}{\sqrt{2}} g_{\zeta}^{s}, \quad g_{a_{0}}^{s}=g_{\sigma}^{s}=g_{\zeta}^{u}=g_{\zeta}^{d}=0, \\
\frac{g_{v}}{2 \sqrt{2}}=g_{\rho^{0}}^{u}=-g_{\rho^{0}}^{d}=g_{\omega}^{u}=g_{\omega}^{d}=\ldots=\frac{1}{\sqrt{2}} g_{\phi}^{s}, \quad g_{\omega}^{s}=g_{\rho^{0}}^{s}=g_{\phi}^{u}=g_{\phi}^{d}=0 .
\end{gathered}
$$

Note, the values of $\sigma_{0}, \zeta_{0}$ and $\chi_{0}$ are determined later from Eqs. (20)-(22). Particularly, the parameters $\sigma_{0}$ and $\zeta_{0}$ are expressed through the pion $\left(F_{\pi}=93 \mathrm{MeV}\right)$ and the kaon $\left(F_{K}=\right.$ $115 \mathrm{MeV})$ leptonic decay constants as:

$$
\sigma_{0}=-F_{\pi} \quad \zeta_{0}=\frac{1}{\sqrt{2}}\left(F_{\pi}-2 F_{K}\right)
$$

The Lagrangian $\mathcal{L}_{\chi S B}$ generates the nonvanishing masses of pseudoscalar mesons

$$
\mathcal{L}_{\chi S B}=\frac{\chi^{2}}{\chi_{0}^{2}}\left[m_{\pi}^{2} f_{\pi} \sigma+\left(\sqrt{2}_{m_{K}}^{2} f_{K}-{\frac{\sqrt{2}^{2}}{m_{\pi}}}_{\pi} f_{\pi}\right) \zeta\right]
$$

leading to a nonvanishing divergence of the axial currents which satisfy the PCAC relations for $\pi$ and $K$ mesons. Scalar mesons obtain the masses by spontaneous breaking of the chiral symmetry in the Lagrangian (8). The masses of $u, d$ and $s$ quarks are generated by the vacuum expectation values of the two scalar mesons $\sigma$ and $\zeta$. To obtain the correct constituent mass of the strange quark, an additional mass term should be added:

$$
\mathcal{L}_{\Delta m_{s}}=-\Delta m_{s} \bar{q} S q
$$

where $S=\frac{1}{3}\left(I-\lambda_{8} \sqrt{3}\right)=\operatorname{diag}(0,0,1)$ is the strangeness quark matrix. Finally, the quark masses are given by

$$
m_{u}=m_{d}=-\frac{g_{s}}{\sqrt{2}} \sigma_{0}, \quad m_{s}=-g_{s} \zeta_{0}+\Delta m_{s}
$$

The parameters $g_{s}=4.76$ and $\Delta m_{s}=29 \mathrm{MeV}$ are determined from $m_{q}=313 \mathrm{MeV}$ and $m_{s}=490 \mathrm{MeV}$. In order to obtain reasonable hyperon potentials in hadronic matter we include the additional coupling between strange quarks and scalar mesons $\sigma$ and $\zeta$ [31. This term is expressed as

$$
\mathcal{L}_{h}=\left(h_{1} \sigma+h_{2} \zeta\right) \bar{s} s
$$

\section{APPLICATION TO STRANGE QUARK MATTER}

Now we apply the model to investigate strange quark matter. We begin with the thermodynamical potential because all other quantities such as energy per volume and pressure can be obtained from it. The thermodynamical potential is defined as

$$
\Omega=\sum_{\tau=q, e} \frac{-2 k_{B} T \gamma_{\tau}}{(2 \pi)^{3}} \int_{0}^{\infty} d^{3} k\left\{\ln \left(1+e^{-\left(E_{\tau}^{*}(k)-\nu_{\tau}\right) / k_{B} T}\right)+\ln \left(1+e^{-\left(E_{\tau}^{*}(k)+\nu_{\tau}\right) / k_{B} T}\right)\right\}-\mathcal{L}_{M}
$$


where $E_{\tau}^{*}(k)=\sqrt{m_{\tau}^{* 2}+k^{2}}, \gamma_{\tau}$ is 3 for quarks and 1 for electrons and $\mathcal{L}_{M}$ is the meson interaction including the scalar meson self-interaction $\mathcal{L}_{\Sigma \Sigma}$, the vector meson self-interaction $\mathcal{L}_{V V}$ and the explicit chiral symmetry breaking term $\mathcal{L}_{\chi S B}$. In the MIT bag and the QMDD model, $\mathcal{L}_{M}$ is replaced by the effective bag constant. At zero temperature, $\Omega$ can be expressed as

$$
\begin{aligned}
\Omega & =-\sum_{i=u, d, s} \frac{1}{8 \pi^{2}}\left\{\nu_{i}\left[\nu_{i}^{2}-m_{i}^{* 2}\right]^{1 / 2}\left[2 \nu_{i}^{2}-5 m_{i}^{* 2}\right]\right. \\
& \left.+3 m_{i}^{* 4} \ln \left[\frac{\nu_{i}+\left(\nu_{i}^{2}-m_{i}^{* 2}\right)^{1 / 2}}{m_{i}^{*}}\right]\right\}-\frac{\mu_{e}^{4}}{12 \pi^{2}}-\mathcal{L}_{M},
\end{aligned}
$$

where $\mu_{e}$ is the chemical potential of the electron and the quantity $\nu_{i}(i=u, d, s)$ is related to the usual chemical potential $\mu_{i}$ by $\nu_{i}=\mu_{i}-g_{\omega}^{i} \omega-g_{\phi}^{i} \phi$. The effective quark mass is given by $m_{i}^{*}=-g_{\sigma}^{i} \sigma-g_{\zeta}^{i} \zeta+m_{i 0}$. The total baryon density is defined as

$$
\rho_{B}=\frac{1}{3}\left(\rho_{u}+\rho_{d}+\rho_{s}\right)
$$

With the thermodynamical potential, the energy per volume $\varepsilon$ and pressure $p$ of the system can be derived as $\varepsilon=\Omega+\sum_{i=u, d, s, e} \mu_{i} \rho_{i}$ and $p=-\Omega$.

The mean field equations for the meson $\phi_{i}$ are obtained with $\frac{\partial \Omega}{\partial \phi_{i}}=0$. For the scalar mesons $\sigma, \zeta$ and $\chi$, the equations are expressed as

$$
\begin{aligned}
& k_{0} \chi^{2} \sigma-4 k_{1}\left(\sigma^{2}+\zeta^{2}\right) \sigma-2 k_{2} \sigma^{3}-2 k_{3} \chi \sigma \zeta-\frac{2 \delta}{3 \sigma} \chi^{4}+\frac{\chi^{2}}{\chi_{0}^{2}} m_{\pi}^{2} f_{\pi}- \\
& -\left(\frac{\chi}{\chi_{0}}\right)^{2} m_{\omega} \omega^{2} \frac{\partial m_{\omega}}{\partial \sigma}=\sum_{i=u, d} g_{\sigma}^{i}<\bar{\psi}_{i} \psi_{i}> \\
& k_{0} \chi^{2} \zeta-4 k_{1}\left(\sigma^{2}+\zeta^{2}\right) \zeta-4 k_{2} \zeta^{3}-k_{3} \chi \sigma^{2}-\frac{\delta}{3 \zeta} \chi^{4}+ \\
& +\frac{\chi^{2}}{\chi_{0}^{2}}\left(\sqrt{2} m_{k}^{2} f_{k}-\frac{1}{\sqrt{2}} m_{\pi}^{2} f_{\pi}\right)=\sum_{i=s} g_{\zeta}^{i}<\bar{\psi}_{i} \psi_{i}> \\
& k_{0} \chi\left(\sigma^{2}+\zeta^{2}\right)-k_{3} \sigma^{2} \zeta+\left(4 k_{4}+1+4 \ln \frac{\chi}{\chi_{0}}-\frac{4 \delta}{3} \ln \frac{\sigma^{2} \zeta}{\sigma_{0}^{2} \zeta_{0}}\right) \chi^{3}+ \\
& +\frac{2 \chi}{\chi_{0}^{2}}\left[m_{\pi}^{2} f_{\pi} \sigma+\left(\sqrt{2} m_{k}^{2} f_{k}-\frac{1}{\sqrt{2}} m_{\pi}^{2} f_{\pi}\right) \zeta\right]-\frac{\chi}{\chi_{0}^{2}} m_{\omega}^{2} \omega^{2}=0
\end{aligned}
$$

The equations for the vector mesons can be obtained in the same way as

$$
\begin{aligned}
\frac{\chi^{2}}{\chi_{0}^{2}} m_{\omega}^{2} \omega+4 g_{4} \omega^{3}+12 g_{4} \omega \rho^{2} & =\sum_{i=u, d} g_{\omega}^{i}<\bar{\psi}_{i} \gamma^{0} \psi_{i}>, \\
\frac{\chi^{2}}{\chi_{0}^{2}} m_{\rho}^{2} \rho+4 g_{4} \rho^{3}+12 g_{4} \omega^{2} \rho & =\sum_{i=u, d} g_{\rho}^{i}<\bar{\psi}_{i} \gamma_{0} \psi_{i}> \\
\frac{\chi^{2}}{\chi_{0}^{2}} m_{\phi}^{2} \phi+8 g_{4} \phi^{3} & =\sum_{i=s} g_{\phi}^{i}<\bar{\psi}_{i} \gamma_{0} \psi_{i}>.
\end{aligned}
$$


The scalar and vector densities can be written as

$$
\begin{aligned}
<\bar{\psi}_{i} \psi_{i}> & =\frac{6}{(2 \pi)^{3}} \int_{0}^{k_{F_{i}}} d^{3} k \frac{m_{i}^{*}}{\sqrt{k^{2}+m_{i}^{* 2}}} \\
<\bar{\psi}_{i} \gamma^{0} \psi_{i}> & =\frac{6}{(2 \pi)^{3}} \int_{0}^{k_{F_{i}}} d^{3} k
\end{aligned}
$$

with $k_{F_{i}}=\sqrt{\nu_{i}^{2}-m_{i}^{* 2}}$.

\section{NUMERICAL RESULTS}

Now we investigate the implications of the previous formalism to strange quark matter. Compared to earlier applications to hadronic matter, here we need not to introduce the confining potential which combines quarks into baryons. In quark matter, the $\mathrm{u}$, $\mathrm{d}$ and $\mathrm{s}$ quarks are deconfined. They only interact by scalar and vector mesons. The self-interactions between mesons are the same as in hadronic matter. All the parameters in this model are determined in our previous papers. They are listed in Table I. We assume that these parameters do not change when the model is applied to quark matter. In fact, the parameters which describe the interactions between fields 'should' be universal. The medium effects are included by the treatment (relativistic mean field theory) itself and are not included in the original Lagrangian. In our discussion, we first do not include the additional coupling $\mathcal{L}_{h}$ and discuss the effect of this term on strange quark matter later.

If strange quark matter is stable and can survive for a long time, equilibrium with respect to the weak processes:

$$
s \rightarrow u+e^{-}+\bar{\nu}_{e}, \quad d \rightarrow u+e^{-}+\bar{\nu}_{e}
$$

may be achieved. If we neglect the chemical potential of the neutrino, the chemical potentials of quarks and electron have the following relation:

$$
\mu_{d}=\mu_{s}=\mu_{u}+\mu_{e}
$$

The values for $\mu_{u}$ and $\mu_{d}$ are determined by the baryon density and the total charge Q. As usually, we assume the charge Q to be zero. The equations for the mesons (20)-(25) can be solved simultaneously. The energy per baryon with different strength of vector coupling and for different parameter sets are shown in Fig.1. When vector interactions are not considered, there is a local minimum of energy per baryon with parameter set A. The corresponding density is about $0.13 \mathrm{fm}^{-3}$. As shown later, at this density no strange quarks appear. When the vector interactions are included, even when the strength is half of that in hadronic matter, the local minimum for the energy per baryon disappears. The energy per baryon $\mathrm{E} / \mathrm{A}$ increases monotonously with the increasing baryon density.

In Fig.2, we show the fractions $r_{i}=\frac{\rho_{i}}{3 \rho_{B}}$ of $\mathrm{u}, \mathrm{d}$, and $\mathrm{s}$ quark versus baryon density with and without vector interactions. The fraction of $u$ quarks almost does not change with the density. There exists a relationship $\rho_{d}+\rho_{s} \simeq 2 \rho_{u}$. Therefore, although we included the electron in our calculations, the fraction of electrons is very small. The chemical potential of the electron $\mu_{e}$ is not zero which results in a larger fraction of $d$ than $u$ quarks. At low 
density no strange quarks are present. The fraction of d quarks is about two times that of $\mathrm{u}$ quarks. Without vector interactions, when the density is larger than about $0.41 \mathrm{fm}^{-3}$, strange quarks appear. Compared to Fig.1, this density is larger than the density where the local minimum appears. This result is close to that of Ref. 30 where the SU(3) NJL model is used. Therefore, no stable strange quark matter can exist at zero pressure in contradiction to the original suggestion by Witten [1] The so-called strange star cannot be composed entirely by the deconfined $\mathrm{u}, \mathrm{d}$ and s quarks. The stable strange quark matter can only exist in the core of these objects where the pressure is high enough to force the transition from hadronic matter to quark matter to occur.

If strange quark matter is metastable, then it may be produced in heavy ion collisions. In this case, the $\beta$-equilibrium may not be achieved. In our calculations, we assume that $\mu_{u}=\mu_{d}=\mu_{q}$. The values of $\mu_{q}$ and $\mu_{s}$ are determined by the total baryon density and the strangeness fraction $f_{s}\left(f_{s}=3 r_{s}\right)$. In Fig.3, we plot the effective masses of nonstrange and strange quarks versus the baryon density for different strangeness fraction with parameter set A. Both $u(d)$ and $s$ quark masses decrease with the increasing density. In nonstrange quark matter, the mass of the $\mathrm{u}(\mathrm{d})$ quark decreases more quickly than that of the s quark. When $f_{s}$ increases, the mass of the s quark decreases at fixed density. At some high density, the strange quark mass is even lower than the mass of nonstrange quarks. Compared to the QMDD model, here the effective quark masses are obtained dynamically. The quark masses are not only density dependent but also strangeness fraction dependent which are not present in the QMDD model.

In Figs.4-6 we plot the energy per baryon versus density for different values of $f_{s}$, which corresponds to different vector coupling constants. The solid and dashed lines are for parameter sets A and B, respectively. First we do not include the interactions between quarks and vector mesons. This is close to the QMDD model or SU(3) NJL model with only scalar type 4-quark interactions. In Fig.4, for parameter set A, there exists a local minimum of energy per baryon for any $f_{s}$. The baryon density at the minimum of energy per baryon first increases and then decreases when $f_{s}$ increases. For nonstrange quark matter, though there is a local minimum, the system has no positive binding energy compared to the vacuum mass of nonstrange quarks. For strange quark matter, the energy per baryon is lower than the masses of hyperons with the same strangeness number. When $f_{s}=1$, the binding energy is about $45 \mathrm{MeV}$ compared to the vacuum constituent quark mass. The maximum binding energy is about $60 \mathrm{MeV}$ and the corresponding $f_{s}$ is about 2.0. Metastable strange quark matter is therefore favored to have a large strangeness fraction (high negative charge). For the parameter set $\mathrm{B}$, the system has a local minimum of energy per baryon only for some range of $f_{s}$, that is $1<f_{s}<3$. The maximum binding energy is about $5 \mathrm{MeV}$, when $f_{s}$ is around 2.0 .

When the interactions between quarks and vector mesons are included, the system is destabilized. In Fig.5, we plot the energy per baryon versus baryon density with the vector coupling constant half of that in hadronic matter. Compared to Fig.4, the energy per baryon becomes higher. For parameter set A, there exists a local minimum for $0<f_{s}<2$. The maximum binding energy is only about $10 \mathrm{MeV}$ with $f_{s} \simeq 1.0$. The corresponding density is also much lower compared to Fig.4. For parameter set B, there is no local minimum for any strangeness fraction. If the vector coupling is the same as in hadronic matter, as is shown in Fig.6, the energy per baryon will increase monotonously with the baryon density for both 
parameter sets $\mathrm{A}$ and $\mathrm{B}$. The vector meson couplings are also discussed in Ref. [29]. Though these two models are quite different, the results are comparable to each other.

If vector interactions are included, even when the strength is only half of that in hadronic matter, the binding energy of metastable strange quark matter becomes small or negative. At the minimum of the energy per baryon, the pressure $\mathrm{p}$ of the system is zero. This kind of objects with finite volume are called strangelets. If the vector interactions are fully considered, the metastable strangelets can not be formed at zero pressure.

Up to now, we did not consider the additional coupling between strange quark and scalar mesons $\mathcal{L}_{h}$, which is important to obtain reasonable hyperon potentials in hadronic matter [31]. When the additional term is included, the effective quark masses versus baryon density for parameter set A are given in Fig.7. The result for the strange quark mass evidently change, especially for large strangeness fraction, when compared to Fig.3. In nonstrange quark matter, the effective mass of s quarks almost stays constant. When the strangeness fraction is high, the strange quark has a lower mass when compared to Fig.3, where $\mathcal{L}_{h}$ is not included

The effective quark masses will affect the energy of the system. In Fig.8 we plot the energy per baryon versus density with the vector coupling constant half of that in hadronic matter. The solid and dashed lines correspond to the cases without and with $\mathcal{L}_{h}$, respectively. For small strangeness fraction $f_{s}$, the results of these two cases are close. For large strangeness fraction, the additional term $\mathcal{L}_{h}$ will produce larger binding energy. If the vector coupling strength is as big as in hadronic matter, there is no local minimum for both cases, with or without $\mathcal{L}_{h}$. Therefore, the inclusion of the additional term $\mathcal{L}_{h}$ does not affect the main results of Fig.1. This is because when the $\beta$-equilibrium is achieved, the strangeness fraction of the system is smaller than 1 . The additional term $\mathcal{L}_{h}$ only gives sizable contributions for systems with a large strangeness fraction.

\section{CONCLUSIONS}

We investigate strange quark matter in a chiral SU(3) quark mean field model. The effective quark masses are obtained dynamically by the quark meson interactions and they are both density and strangeness fraction dependent. The stability of strange quark matter is studied for different values of the vector coupling constant and for different parameter sets. The effect of the additional term $\mathcal{L}_{h}$ is also discussed.

If the strange quark is stable and can survive a long time, the $\beta$-equilibrium can be achieved. The strangeness fraction $f_{s}$ is smaller than 1. In the chiral $\mathrm{SU}(3)$ quark model, at the density where the system has a local minimum for the energy per baryon, no strange quarks appear. Even without the vector meson coupling, this nonstrange quark matter has a negative binding energy and cannot be bound. Therefore, opposed to the suggestion by Witten, stable quark matter at zero pressure cannot exist even without vector meson interactions.

If quark matter can be produced in heavy ion collisions, the $\beta$-equilibrium may not be achieved. This metastable strange quark matter can have a high strangeness fraction (high negative charge). When we do not take the vector meson interaction into account, as in the QMDD model or SU(3) NJL model with only scalar type 4-quark interaction, the maximum binding energy is about $5 \mathrm{MeV}-60 \mathrm{MeV}$ and the corresponding strangeness fraction is 
about 1 - 2. If we assume that the strength of vector meson coupling is half of that in hadronic matter (in this case, the strength of vector coupling is comparable to the one of Ref. [29]), the maximum binding energy decreases. Inclusion of the additional term $\mathcal{L}_{h}$ will forces the system to have a larger strangeness fraction and binding energy. When the vector coupling constant is the same as for hadronic matter, no metastable strange quark matter (strangelets) can be formed in heavy ion collisions.

However, strange quark matter can still exist in the core of a neutron star. The phase transition from hadronic matter to quark matter can occur at high density and pressure which is caused by gravity. Because both hadronic and quark matter can be described in the $\mathrm{SU}(3)$ quark mean field model, it is of interest to investigate this phase transition in this model. This will be studied in the future.

Acknowledgements. P. Wang would like to thank the Institute for Theoretical Physics, University of Tübingen for their hospitality. This work was supported by the Alexander von Humboldt Foundation and by the Deutsche Forschungsgemeinschaft (DFG) under contracts FA67/25-1, GRK683. 


\section{REFERENCES}

[1] E. Witten, Phys. Rev. D 30, 272 (1984).

[2] M. Kasuya et al., Phys. Rev. D 47, 2153 (1993).

[3] M. Ichimura et al., Nuovo Cim. A 106, 843 (1993).

[4] J. N. Capdevielle et al., Nuovo Cim. C 19, 623 (1996).

[5] I. Bombaci, Phys. Rev. C 55, 1587 (1997).

[6] K. S. Cheng, Z. G. Dai, D. M. Wai, and T. Lu, Science 280, 407 (1998).

[7] M. Dey, I. Bombaci, J. Dey, S. Ray, and B. C. Samanta, Phys. Lett. B 438, 123 (1998); Erratum Phys. Lett. B 467, 303 (1999).

[8] X. D. Li, I. Bombaci, M. Dey, J. Dey, and E. P. J. van den Heuvel, Phys. Rev. Lett. 83, 3776 (1999).

[9] C. Greiner, P. Koch, and H. Stöcker, Phys. Rev. Lett. 58, 1825 (1987).

[10] C. Greiner, D. H. Rischke, H. Stöcker, and P. Koch, Phys. Rev. D 38, 2797 (1988).

[11] C. Greiner, J. Schaffner, and H. Stöcker, Nucl. Phys. Proc. Suppl. B 24, 239 (1991).

[12] J. Barrette et al., Phys. Lett. B 252, 550 (1990); M. Aoki et al., Phys. Rev. Lett. 69, 2345 (1992); K. Borer et al., ibid. 72, 1415 (1994); D. Beavis et al., ibid 75, 3078 (1995).

[13] D. Ardouin et al., Phys. Lett. B 446, 191 (1999).

[14] T. A. Armstrong et al., Phys. Rev. C 59, 1829 (1999); Phys. Rev. C 63054903 (2001).

[15] A. Chodos et al., Phys. Rev. D 9, 3472 (1974).

[16] E. Farhi and R. L. Jaffe, Phys. Rev. D 30, 2379 (1984).

[17] M. S. Berger and R. L. Jaffe, Phys. Rev. C 35, 213 (1987).

[18] J. Madsen, Phys. Rev. Lett. 70, 391 (1993); Phys. Rev. D 47, 5156 (1993).

[19] A. Ukawa, Nucl. Phys. A 498, 227c (1989).

[20] G. N. Fowler, S. Raha, and R. M. Weiner, Z. Phys. C 9, 271 (1981).

[21] S. Chakrabarty, S. Raha, and B. Sinha, Phys. Lett. B 229, 112 (1989).

[22] S. Chakrabarty, Phys. Rev. D 43, 627 (1991).

[23] S. Chakrabarty, Phys. Rev. D 48, 1409 (1993).

[24] O. G. Benvenuto and G. Lugones, Phys. Rev. D 51, 1989 (1995).

[25] G. X. Peng, H. C. Chiang, P. Z. Ning, and B. S. Zou, Phys. Rev. C 59, 3452 (1999).

[26] P. Wang, Phys. Rev. C 62, 015204 (2000).

[27] W. M. Alberico, A. Drago, and C. Ratti, hep-ph/0110091.

[28] M. Jaminon and B. Van den Bossche, Nucl. Phys. A 686, 341 (2001).

[29] I. N. Mishustin, L. M. Satarov, H. Stöcker, and W. Greiner, Phys. Atom. Nucl. 64, 802 (2001).

[30] M. Buballa and M. Oertel, Phys. Lett. B 457, 261 (1999).

[31] P. Wang, Z. Y. Zhang, Y. W. Yu, R. K. Su, and H. Q. Song, Nucl. Phys. A 688, 791 (2001).

[32] P. Wang, H. Guo, Z. Y. Zhang, Y. W. Yu, R. K. Su, and H. Q. Song, Nucl. Phys. A 705, 455 (2002).

[33] P. Papazoglou, D. Zschiesche, S. Schramm, J.Schaffner-Bielich, H. Stöcker, and W. Greiner, Phys. Rev. C 59, 411 (1999). 


\section{TABLES}

TABLE I. Parameters of sets A and B of the model.

\begin{tabular}{c|c|c|c|c|c|c|c|c|c|c|}
\hline \hline set & $k_{0}$ & $k_{1}$ & $k_{2}$ & $k_{3}$ & $k_{4}$ & $g_{s}$ & $g_{v}$ & $g_{4}$ & $h_{1}$ & $h_{2}$ \\
\hline A & 4.94 & 2.12 & -10.16 & -5.38 & -0.06 & 4.76 & 10.92 & 37.5 & -2.20 & 3.24 \\
B & 3.83 & 2.64 & -10.16 & -3.40 & -0.18 & 4.76 & 10.13 & 0.0 & -2.03 & 2.55 \\
\hline \hline
\end{tabular}

Figure captions

Fig.1: Energy per baryon versus baryon density for different vector coupling constants. The solid and dashed lines are for parameter sets A and B. Results are shown for the case of $\beta$-equilibrium.

Fig.2: Fractions of $\mathrm{u}, \mathrm{d}$ and s quarks in strange matter versus baryon density with and without vector interactions, respectively. The solid and dashed lines are for parameter sets $\mathrm{A}$ and B. Results are shown for the case of $\beta$-equilibrium.

Fig.3: The effective nonstrange and strange quark mass versus baryon density for different strangeness fraction. Parameters are taken from set A.

Fig.4: The energy per baryon versus baryon density for different strangeness fraction for parameter sets $\mathrm{A}$ and $\mathrm{B}$. The interaction between quarks and vector mesons is not considered.

Fig.5: Same as in Fig. 4, but here the quark-vector meson interaction is included. The value of the vector coupling constant is half of that used in hadronic matter.

Fig.6: Same as Fig. 5, but the vector coupling constant is of the strength as in hadronic matter.

Fig.7: Same as Fig. 3, but the the additional term $\mathcal{L}_{h}$ is included in the calculation.

Fig.8: The energy per baryon versus baryon density calculated with parameter set A. The vector coupling constant is half of that in hadronic matter. Solid and dashed lines correspond to the case without and with the additional term $\mathcal{L}_{h}$, respectively. 


\section{FIGURES}

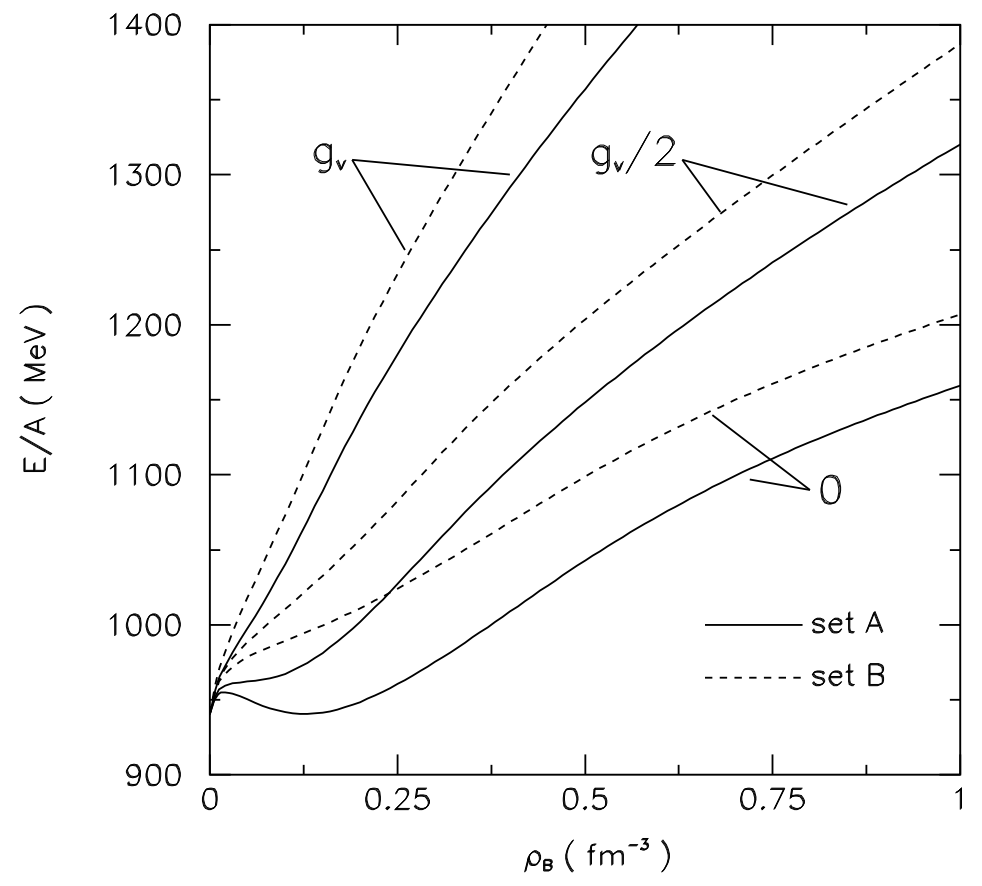

Fig.1 


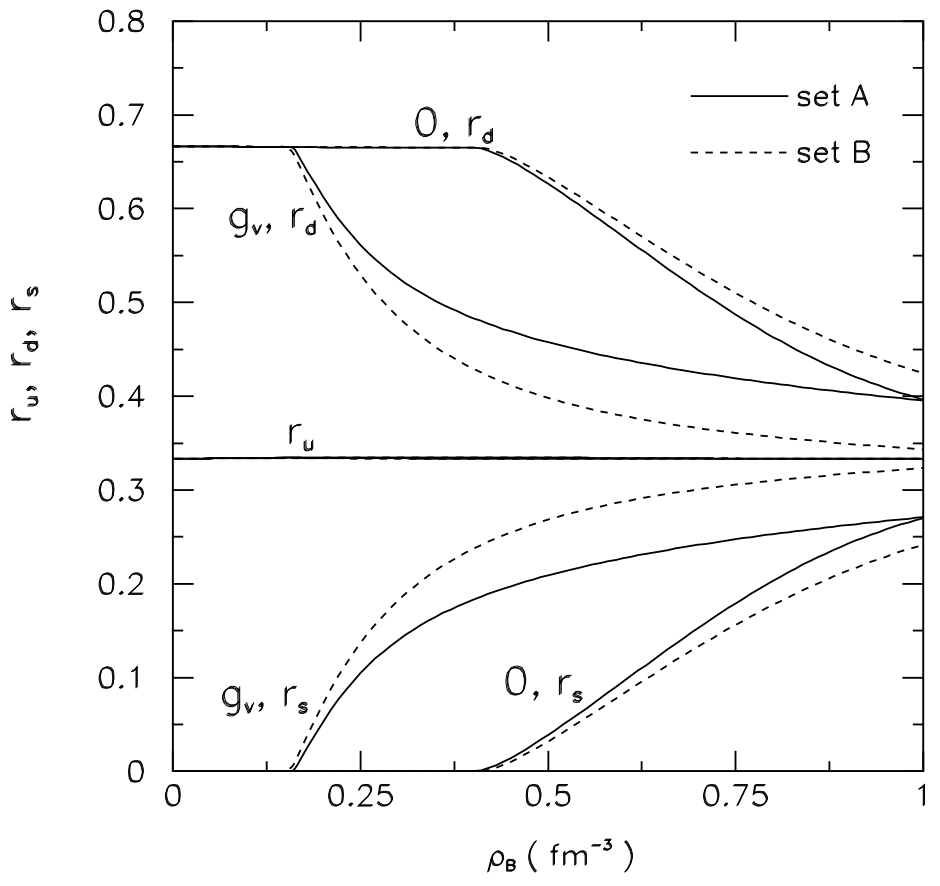

Fig.2 


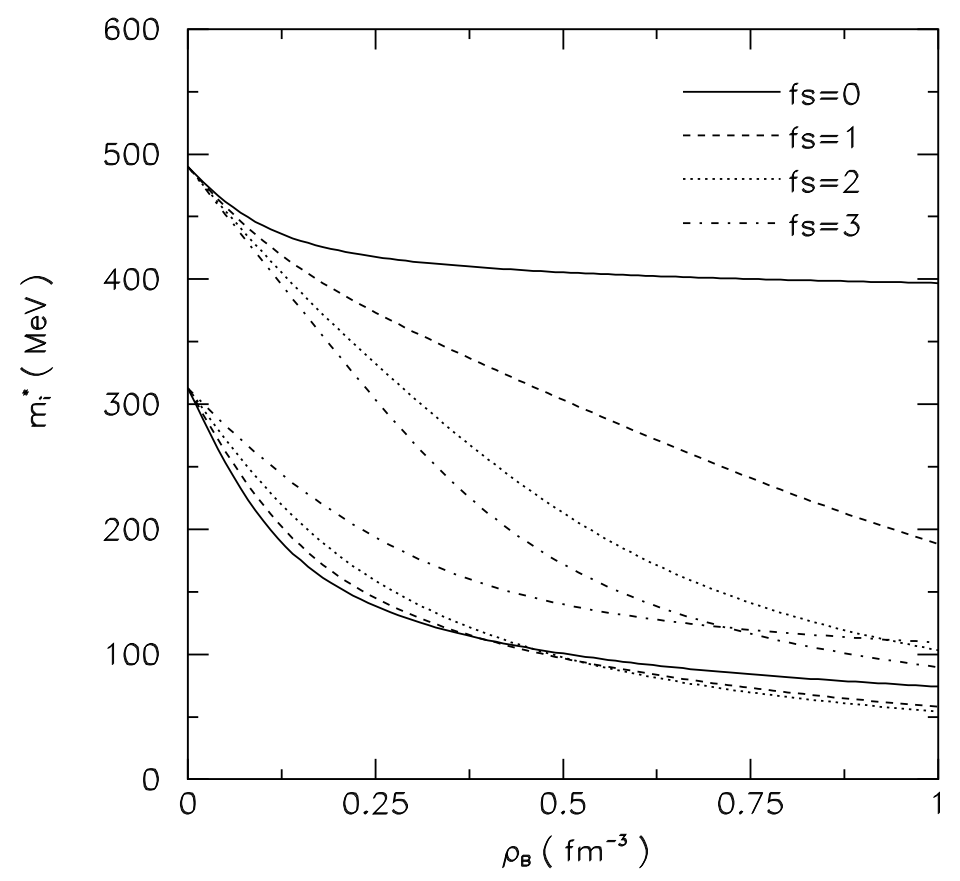

Fig.3 


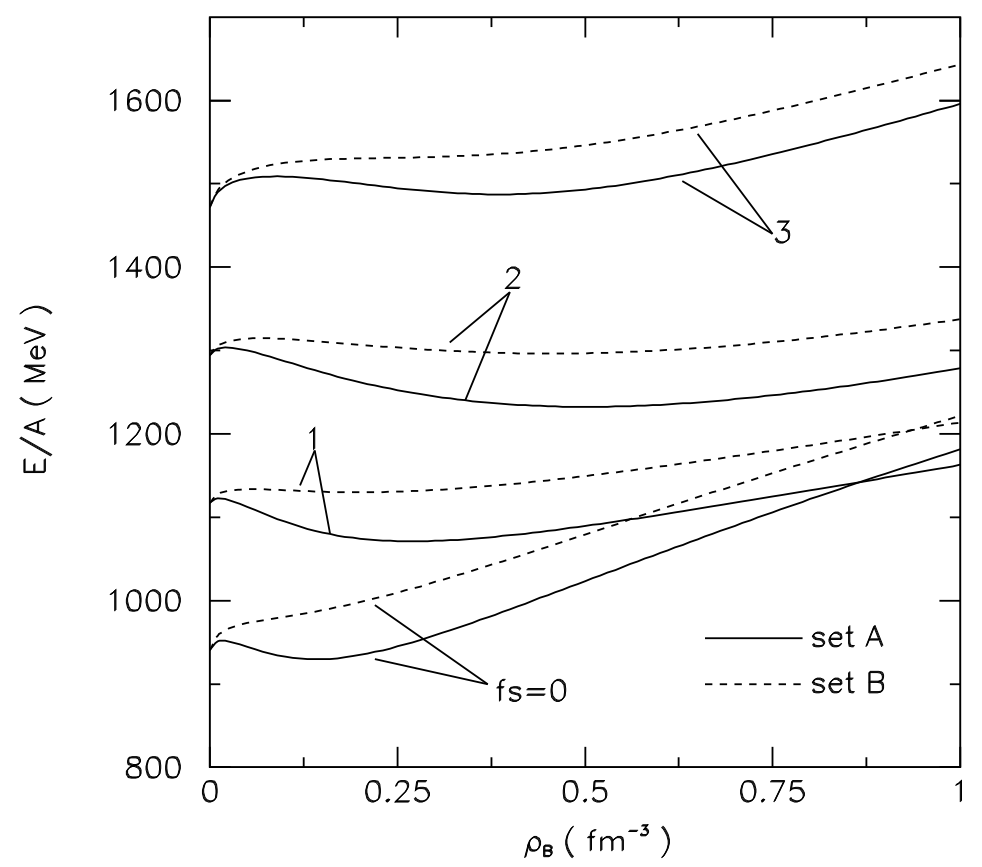

Fig.4 


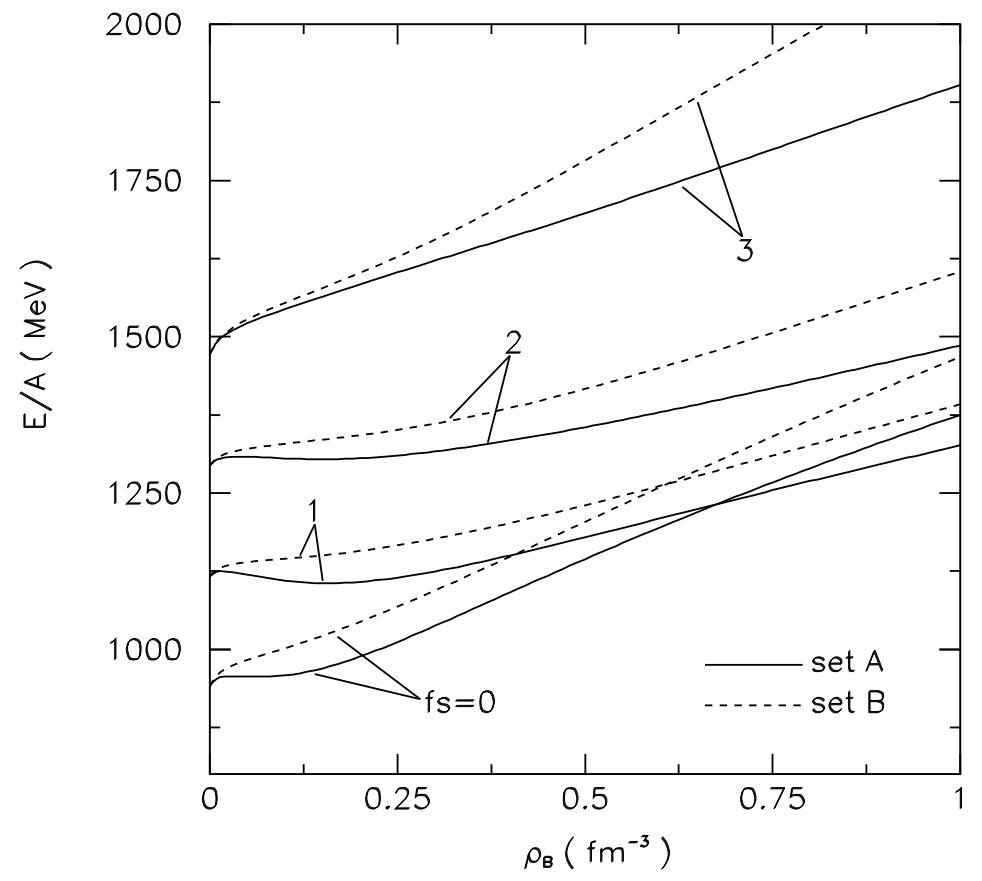

Fig.5 


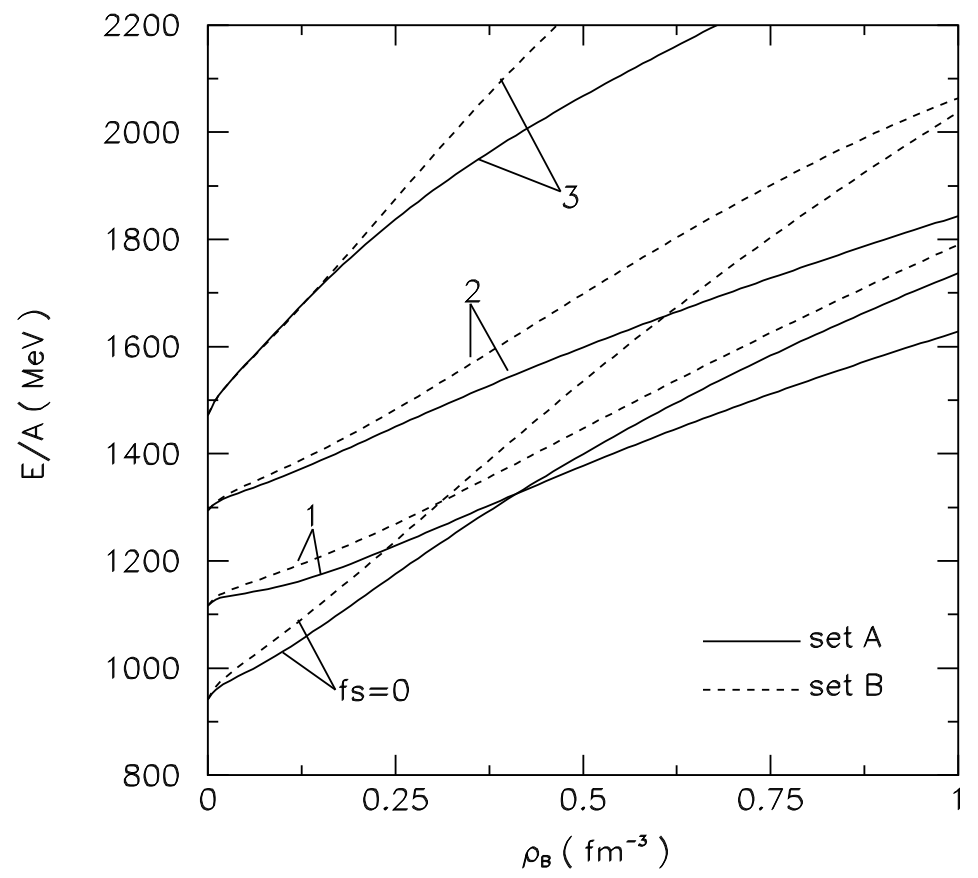

Fig.6 


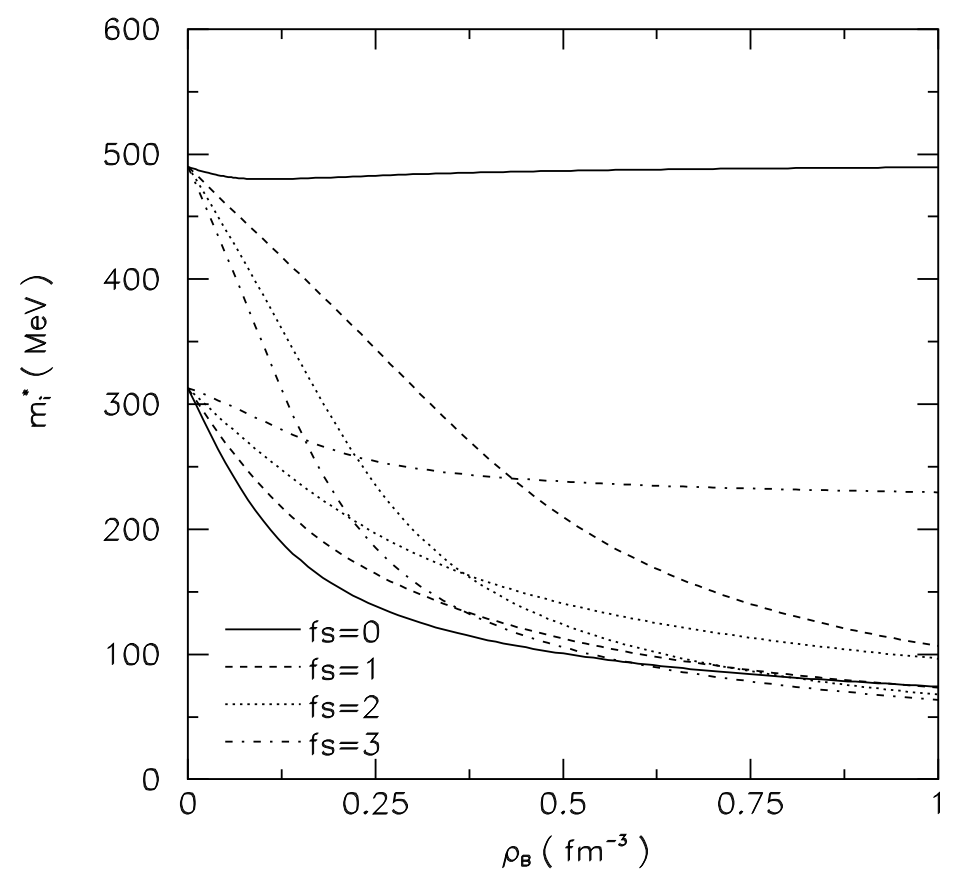

Fig.7 


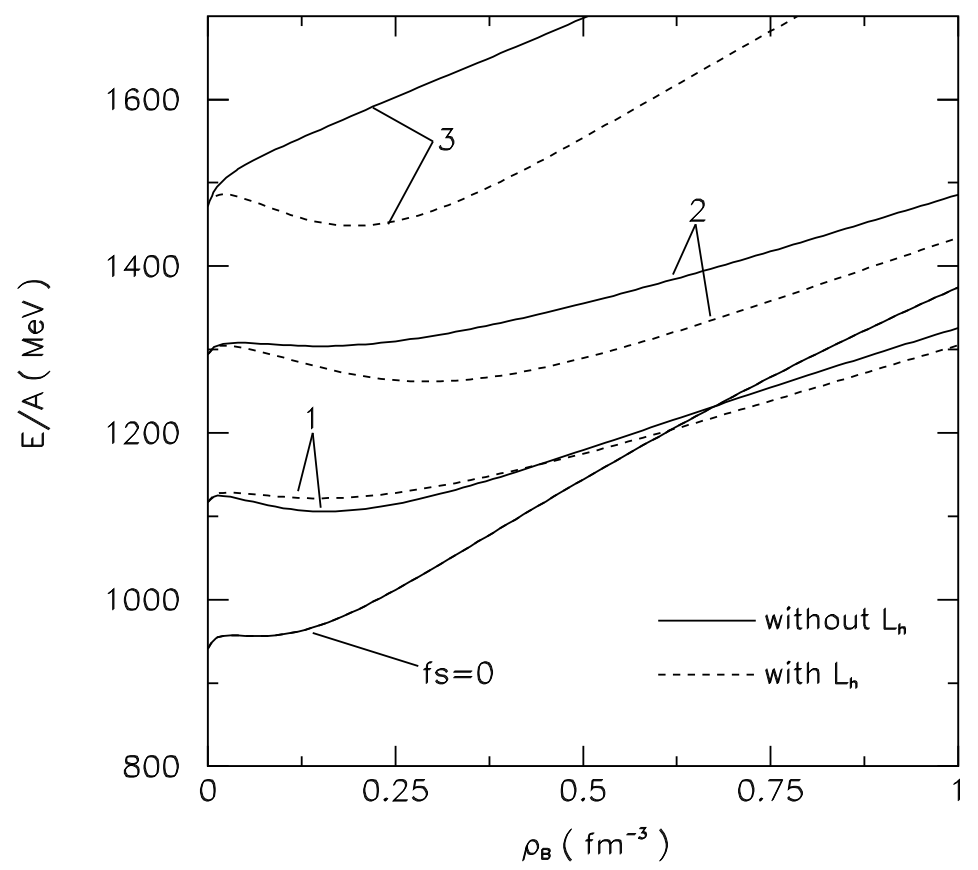

Fig.8 